\title{
Erratum to: Viscosity-mediated motion coupling between pairs of trichobothria on the leg of the spider Cupiennius salei
}

\author{
Brice Bathellier • Friedrich G. Barth • \\ Jörg T. Albert · Joseph A. C. Humphrey
}

Published online: 4 December 2009

(C) Springer-Verlag 2009

\section{Erratum to: J Comp Physiol A (2005) 191:733-746 DOI 10.1007/s00359-005-0629-5}

It was recently pointed out to us that Figs. 5-8 in this article could not be correctly reproduced with the equations provided. As it turned out, the problem is due to an incorrect transcription of the final version of the manuscript to the printed text in regard to Eq. 21 in the Appendix, and the terms given in the third column of Table 1. These typographic errors, however, do not affect our results and conclusions in any way.

1. In Eq. 21 of the Appendix, two minus signs were omitted. The correct equation reads:
The online version of the original article can be found under doi:10.1007/s00359-005-0629-5.

\section{Present Address:}

B. Bathellier ( $\square)$

Research Institute of Molecular Pathology (IMP),

Dr. Bohr-Gasse 7, 1030 Wien, Austria

B. Bathellier · F. G. Barth · J. T. Albert

Department of Neurobiology and Cognition Research,

Life Sciences, University of Vienna, Althanstr. 14,

1090 Wien, Austria

e-mail: friedrich.g.barth@univie.ac.at

\section{J. A. C. Humphrey}

Department of Mechanical and Aerospace Engineering,

University of Virginia, Charlottesville, VA 22904, USA

$$
\begin{aligned}
D_{x}= & \frac{v_{\infty}-v_{x}^{*}}{v_{\infty}-v_{h}^{*}} \\
= & -\left(\frac{K_{2}(\lambda)}{K_{0}(\lambda)} \frac{1}{4 \eta^{2}}+\frac{2 K_{1}^{\prime}(2 \lambda \eta)}{K_{0}(\lambda)}\right) \sin ^{2}(\Psi) \\
& +\left(\frac{K_{2}(\lambda)}{K_{0}(\lambda)} \frac{1}{4 \eta^{2}}-\frac{K_{1}(2 \lambda \eta)}{\lambda \eta K_{0}(\lambda)}\right) \cos ^{2}(\Psi)
\end{aligned}
$$

with $\lambda=\frac{1+i}{\sqrt{2}} \frac{d}{2 \xi}=\frac{1+i}{\sqrt{2}} \frac{d}{2} \sqrt{\frac{2 \pi f}{v}}$, where $\eta=r / d, r$ is the distance from the origin, $d$ is the hair diameter, $v$ is the kinematic viscosity of air, and $f$ is the frequency of the oscillating air flow.

2. On p. 740, at the end of the first column, the parameter $G$ is erroneously given as $G=g /\left(g^{2}+\pi^{2} / 16\right)$ when it should be $G=-g /\left(g^{2}+\pi^{2} / 16\right)$.

3. In Table 1, the $D_{0}$ terms in the numerators of the terms shown in the 4th column were erroneously raised to the power of 2. In addition, $L_{1}$ and $L_{2}$ were erroneously interchanged in all terms of row 3 and the last term of row 4 . The correct Table 1 reads:

\begin{tabular}{llll}
\hline & $\varepsilon^{\prime}$ & $\varepsilon^{\prime \prime}$ & $\varepsilon^{\prime \prime \prime}$ \\
\hline$L_{1}=L_{2}$ & $1-\frac{D_{0}}{1+D_{0}}$ & $1+\frac{D_{0}^{2}}{1-D_{0}^{2}}$ & $\frac{D_{0}}{1-D_{0}^{2}}$ \\
$L_{1}>L_{2}$ & $1-\frac{L_{2}^{2}}{L_{1}^{2}} \frac{D_{0}}{1+D_{0}}$ & $1+\frac{L_{2}^{3}}{L_{1}^{3}} \frac{D_{0}^{2}}{1-D_{0}^{2}}$ & $\frac{L_{2}^{2}}{L_{1}^{2}} \frac{D_{0}}{1-D_{0}^{2}}$ \\
$L_{1}<L_{2}$ & $1-\frac{D_{0}}{1+D_{0}}$ & $1+\frac{D_{0}^{2}}{1-D_{0}^{2}}$ & $\frac{L_{1}}{L_{2}} \frac{D_{0}}{1-D_{0}^{2}}$ \\
\hline
\end{tabular}

4. In Fig. 5, the air flow is directed along the $x$ axis and not along the $y$ axis as stated in the figure caption.

5. The coupling coefficient $\kappa_{1}$ for Hair 1 , defined as $\kappa_{1}=\left(\theta_{\text {ref1 }}-\theta_{1}\right) / \theta_{\text {ref1 }}$ and plotted in Figs. 6 and 7, is not valid for the case of Hair 2 being mechanically driven. In this case, $\kappa_{1}=\theta_{1} / \theta_{2}$. 